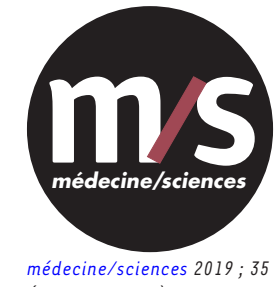

(hors série $n^{\circ} 1$ ) : 35-6

\section{Anticiper le changement et les risques chez l'adulte}

De I'AMM à l'utilisation d'un traitement innovant : quel parcours ! (2)

Jean-Christophe Antoine

\begin{abstract}
$>$ Utiliser des médicaments novateurs en post-autorisation de mise sur le marché chez l'adulte ou chez l'enfant ne présente pas de différence, si ce n'est quantitative. Les adultes représentent en effet près de $80 \%$ des patients atteints de maladies neuromusculaires. Leur prise en charge thérapeutique nécessitera donc de changer d'échelle, une nouvelle donne aux implications multiples. <
\end{abstract}

Les consultations et services qui accueillent des adultes atteints de maladies neuromusculaires ont déjà une expérience de traitements innovants, à l'exemple des biothérapies utilisées depuis longtemps déjà pour les pathologies dysimmunitaires. Dans le cadre des maladies génétiques, un traitement innovant ne relève pas toujours de la thérapie génique. II peut s'agir de chimiothérapie classique. De nombreux essais cliniques sont en cours avec des molécules connues et anciennes, notamment dans la maladie de Charcot-Marie-Tooth. Si leurs résultats s'avèrent positifs, nous pouvons imaginer que la mise en œuvre de ces traitements soit beaucoup plus simple que celle d'une biothérapie. Deux thérapies géniques ont obtenu une autorisation de mise sur le marché (AMM) chez l'adulte, toute deux pour la neuropathie amyloïde héréditaire liée à la transthyrétine de l'adulte. II s'agit du patisiran (Onpattro ${ }^{\circledR}$ ) et de l'inotersen (Tegsedi ${ }^{\circledR}$ ). L’Agence européenne du médicament (European Medicines Agency ou EMA) leur a accordé une AMM en août 2018. C'est très récent. Tout reste donc à construire pour les maladies génétiques de l'adulte. Comment faire face? Comment se préparer à délivrer ces nouvelles thérapies? Tout dépendra du traitement, de son mode d'administration, simple sous forme de comprimés ou plus complexe avec des injections, et des manipulations nécessaires, totalement différentes si la thérapie utilise un vecteur viral.

\section{Se former, s'organiser... et financer !}

Les équipes de soins vont devoir être formées. Actuellement, leur formation aux traitements innovants repose sur la seule industrie pharmaceutique. Les laboratoires sont certes bien placés pour donner des conseils sur leurs médicaments, mais d'autres acteurs pourraient intervenir. Les filières de santé maladies rares, comme Filnemus pour les maladies neuromusculaires, ont à mon sens un rôle à jouer. Elles pourraient participer

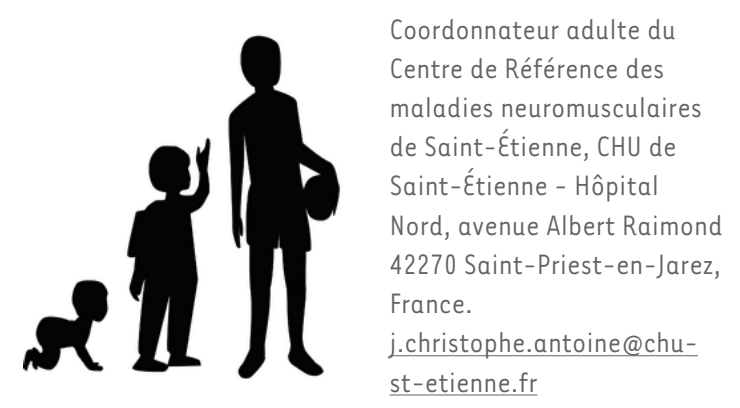

à la formation du personnel, ne serait-ce qu'en créant des espaces professionnels où échanger des questions/ réponses sur des thèmes d'ordre purement pratique, certainement très utiles. L'arrivée des thérapies innovantes chez l'adulte soulève également des problématiques organisationnelles et financières. Ces thérapeutiques assez spécifiques vont nécessiter des conditions d'administration dans certains cas assez complexes. Or la prise en charge des patients dans nos structures hospitalières s'envisage de plus en plus en ambulatoire, en hôpital de jour multi-spécialités, où un patient atteint d'une maladie neuromusculaire va éventuellement côtoyer un patient venu pour une pathologie aiguë et de courte durée. Difficile d'imaginer qu'un traitement innovant et complexe puisse se faire dans de telles structures partagées. II y aura là certainement un combat à mener auprès de nos administrations pour leur faire comprendre la nécessité de dérogations à la règle générale. Tout dépendra du personnel nécessaire. Mettre en place des thérapeutiques et former les équipes va prendre du temps. Administrer les traitements également. Le dimensionnement du financement du $3^{e}$ Plan national maladies rares, soit près 780 millions d'euros [1], sera-t-il suffisant pour disposer des ressources paramédicales et médicales nécessaires à la prise en charge des patients adultes? Ce n'est pas certain en raison d'un « effet de nombre » aux conséquences encore difficiles à évaluer. Huit patients atteints d'une maladie neuromusculaire sur dix sont en effet un adulte. 
Imaginez par exemple un traitement innovant pour une pathologie comme la maladie de Steinert, dont la prévalence est estimée à 12,5 pour 100 000 [2]. L'arrivée de traitement innovant s'accompagne également de la nécessité d'établir des registres de suivi des patients traités. Les remplir prend, là encore, du temps. Et les créer coûte cher, d'autant qu'il va falloir en établir un pour chaque maladie, si ce n'est pour chaque traitement. Le financement de ces registres pourrait s'appuyer sur l'industrie pharmaceutique, avec laquelle il va falloir négocier, négociations dans lesquelles la filière Filnemus pourrait s'impliquer. Le choix du contenu des registres devra toutefois rester à la main des cliniciens.

\section{Un danger de concentration}

In fine se profile pour demain, encore une fois en raison du nombre d'adultes atteints de maladies neuromusculaires, un risque très français: le centralisme. Nos administrations pourraient décider que tel traitement sera administré à tel endroit, qui sera doté des moyens adéquats. Cette issue pourrait relever d'une décision administrative franche ou d'un processus bien plus insidieux, au fil duquel les structures les moins bien dotées finiront par se trouver dans l'incapacité d'assumer la charge de travail que représentent les traitements innovants. Une telle centralisation serait préjudiciable aux patients, obligés de se rendre dans la structure élue pour recevoir leur traitement, souvent itératif. Ce serait une perte de chance pour nombre d'entre eux. La centralisation serait également préjudiciable au progrès thérapeutique. Les techniques sur lesquelles s'appuient les médicaments innovants des maladies neuromusculaires, et notamment la thérapie génique, pourraient en effet être appliquées à d'autres pathologies beaucoup plus fréquentes comme les cancers ou la maladie d'Alzheimer. Un système trop centralisé freinerait leur développement et leur diffusion en France. $\diamond$

From label to delivery of an innovative treatment: what a journey! (2)

Anticipating changes and risks in adults

\section{LIENS D’INTÉRÊT}

L'auteur déclare n'avoir aucun lien d'intérêt concernant les données publiées dans cet article.

\section{RÉFÉRENCES}

1. Plan national maladies rares 2018-2022 - Partager l'innovation, un diagnostic et un traitement pour chacun. Paris, France : Ministère des Solidarités et de la Santé (sur http://solidarites-sante.gouv.fr).

2. Prévalence des maladies rares: données bibliographiques. Les Cahiers $d^{\prime}$ Orphanet, série Maladies rares $n^{\circ} 1$, juin 2018 (sur http://www.orpha.net).

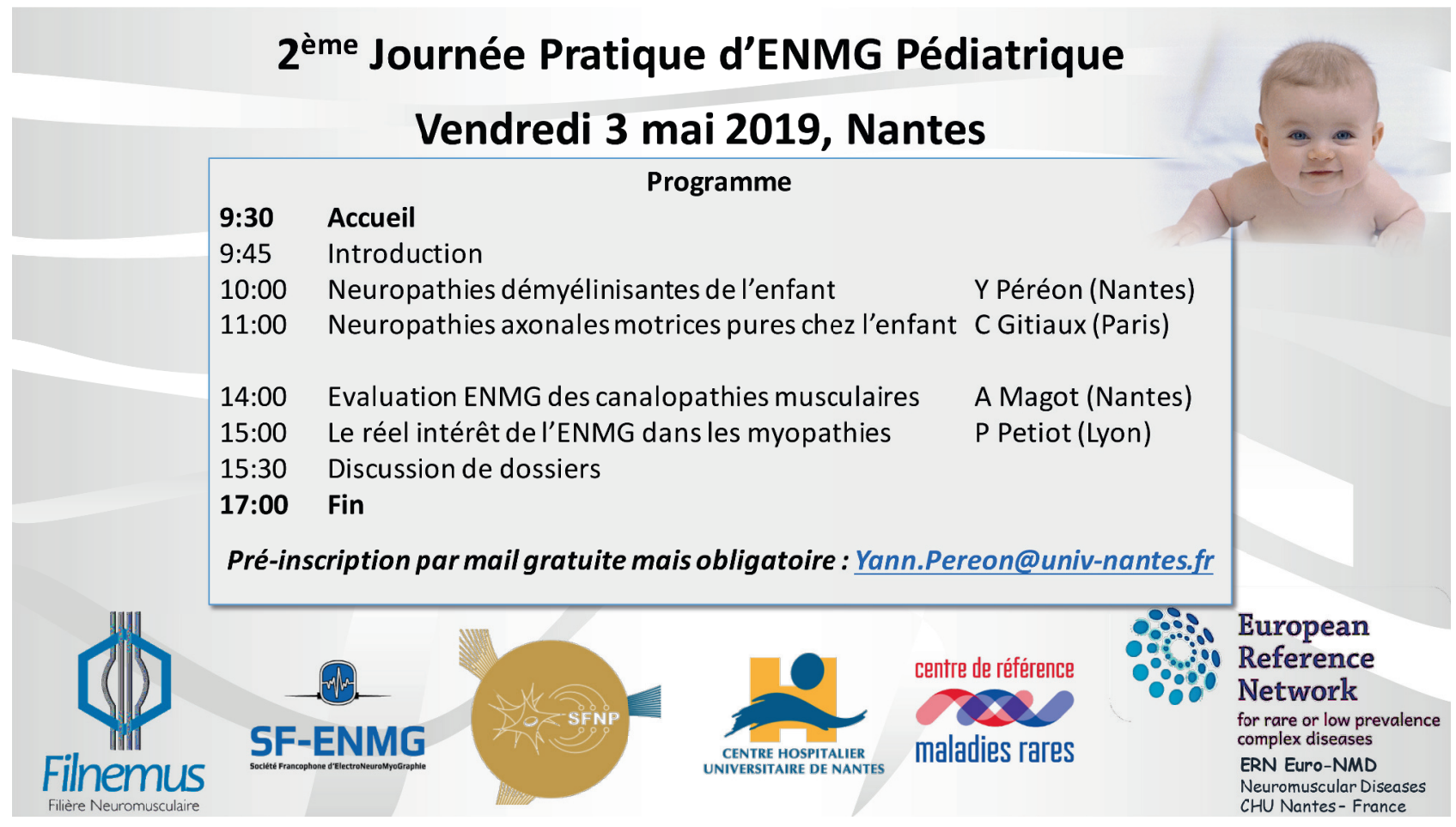

